\title{
Filigrane
}

Écoutes psychothérapiques

\section{"Père, ne vois-tu pas que je suis malade ? " Au nom de mon frère mort, je demande la vie}

\section{Céline Masson}

Volume 19, numéro 1, printemps 2010

Adieu Oedipe, bonjour Narcisse ?

URI : https://id.erudit.org/iderudit/044181ar

DOI : https://doi.org/10.7202/044181ar

Aller au sommaire du numéro

Éditeur(s)

Revue Santé mentale au Québec

ISSN

1192-1412 (imprimé)

1911-4656 (numérique)

Découvrir la revue

Citer cet article

Masson, C. (2010). « Père, ne vois-tu pas que je suis malade ? » Au nom de mon frère mort, je demande la vie. Filigrane, 19(1), 77-88.

https://doi.org/10.7202/044181ar

\section{Résumé de l'article}

À partir du cas de Jérôme, père de Denis venant en thérapie pour comprendre les difficultés de son lien à son fils, vivant en grande précarité, désinséré, en retrait de toute vie sociale et professionnelle, nous avons souhaité réinterroger les modes de nouage du lien père-fils et la possibilité pour un fils de prendre un nom du père sans en être aliéné : comme disait Lacan on peut se passer du Nom-du-Père à condition de s'en servir. Denis est écrasé par l'uniforme d'un père qu'il voit comme tout-puissant et castrateur (rêve du taureau). L'occasion nous est donnée alors par ce rêve de Denis rapporté à son père et que ce dernier nous rapporte en séance, de revenir à Totem et tabou de Freud et à la fonction du père dans le système de la horde primitive. Le père est tué in effigie, en image, on brise sa statue au risque de ne plus exister soi-même. Le mythe de la horde primitive de Totem et tabou est bien un mythe. Le meurtre du père est un passage obligé pour tout sujet quand il doit s'inscrire dans le groupe social. Ce cas clinique n'est pas sans nous évoquer la Lettre au père de Kafka et le poids du père qui l'écrase et dont l'écriture va être une tentative de se faire un nom en propre et se tirer d'affaire. 


\title{
«Père, ne vois-tu pas que je suis malade?" Au nom de mon frère mort, je demande la vie
}

\author{
Céline Masson
}

\begin{abstract}
À partir du cas de Jérôme, père de Denis venant en thérapie pour comprendre les difficultés de son lien à son fils, vivant en grande précarité, désinséré, en retrait de toute vie sociale et professionnelle, nous avons souhaité réinterroger les modes de nouage du lien père-fils et la possibilité pour un fils de prendre un nom du père sans en être aliéné : comme disait Lacan on peut se passer du Nom-du-Père à condition de s'en servir. Denis est écrasé par l'uniforme d'un père qu'il voit comme tout-puissant et castrateur (rêve du taureau). L'occasion nous est donnée alors par ce rêve de Denis rapporté à son père et que ce dernier nous rapporte en séance, de revenir à Totem et tabou de Freud et à la fonction du père dans le système de la horde primitive. Le père est tué in effigie, en image, on brise sa statue au risque de ne plus exister soi-même. Le mythe de la horde primitive de Totem et tabou est bien un mythe. Le meurtre du père est un passage obligé pour tout sujet quand il doit s'inscrire dans le groupe social. Ce cas clinique n'est pas sans nous évoquer la Lettre au père de Kafka et le poids du père qui l'écrase et dont l'écriture va être une tentative de se faire un nom en propre et se tirer d'affaire.
\end{abstract}

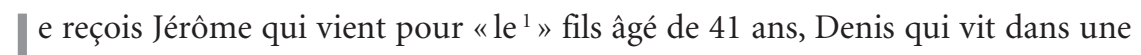
grande précarité, sans emploi et vraisemblablement en grande souffrance psychique (dépression). Jérôme, à la retraite maintenant, souhaite que je l'aide à se rapprocher de son fils afin de parvenir lui-même à l'aider. Son désarroi m’interpelle et m'engage à tenter avec lui un travail. Nous nous verrons chaque semaine pendant six mois environ, puis Jérôme fait un accident vasculaire qui interrompra les séances, même après sa sortie de l'hôpital.

Afin de suivre le déroulement des séances que je propose de présenter, je dois préciser que je ne vois que le père, que j'appelle Jérôme, qui expose de séance en séance l'histoire de son fils Denis. Toutefois le père raconte à son fils, au bout de quelques séances, qu'il vient me voir pour lui et qu'il espère que leur relation va ainsi s'améliorer. Jérôme me rapporte donc au fil du temps les propos de Denis, qui réagit aussi aux interventions que je peux faire suite aux rencontres avec son père. Il se tisse alors une thérapie à trois, dont l'un suit au fil (au téléphone) la manière dont se déroulent les séances de son père avec moi. J'ai effectivement la sensation d'être prise dans un dispositif que nous inventons de semaine en semaine, en laissant place à 
l'absent (le fils). À plusieurs reprises je me suis posée la question de la place que j'occupais : thérapeute de qui ? Du père, du fils? Qui suis-je pour Jérôme? Sa thérapeute? Celle de son fils? Je ne peux parler de thérapie familiale, qui requiert la présence des membres de la famille concernés in situ, mais d'une thérapie impliquant un membre de la famille à distance, le père me rapportant les propos dont son fils lui fait part pour la séance.

«Les fils qui sont entre nous sont ténus », «ça pourrait se rompre». Le père apparaît comme un père rigide (nous verrons plus loin qu'il n'en est rien), ayant pratiqué une éducation exigeante. Il a bâti une grande famille (comme d'ailleurs a-t-il construit de grands projets dans les règles de l'art), cinq enfants dont l'un est mort à l'âge de quatre ans, deux ans avant la naissance de Denis. La fratrie est composée de trois filles et ce fils venant en troisième position. Jérôme dit venir sur les conseils de sa femme qui est très inquiète pour son fils et qui souhaiterait aussi me voir. Je ne la verrai finalement pas.

«Je suis malade» dit Denis à son père au fil ; le père lui signifie qu'il doit donc voir un médecin s'il est malade. D'où a-t-il mal? D’une parole impossible, de fils noués entre père et fils, ce qui n'est peut-être pas sans lien avec cet enfant mort. Jérôme me dit avoir beaucoup attendu de Denis, car il est intelligent et aurait pu faire de grandes choses (comme lui). Ce fils n'a cessé de se faire renvoyer des lieux où il a été «inscrit» et même de chez les Jésuites. Être inscrit et tenir le lieu, voilà ce à quoi Denis s'efforcera de ne pas tenir. «Ça il savait faire, au moins il a montré qu’il était capable de quelque chose... se faire renvoyer.»

Quelle est la brûlure de ce fils? Je fais en effet allusion au rêve que rapporte Freud dans sa Traumdeutung. Ce rêve lui est raconté par une patiente qui le tient elle-même d'une conférence sur le rêve à la suite de laquelle elle s'empressa de le rêver à son tour. «Un père a veillé jour et nuit, pendant longtemps, auprès du lit de son enfant malade. Après la mort de l'enfant, il va se reposer dans une chambre à côté [...]. Un vieillard a été chargé de la veillée mortuaire, il est assis auprès du cadavre et marmotte des prières. Au bout de quelques heures de sommeil, le père rêve que l'enfant est près de son lit, lui prend le bras, et murmure d'un ton plein de reproche: « $\mathrm{Ne}$ vois-tu donc pas que je brûle?». Il s'éveille, aperçoit une vive lumière provenant de la chambre mortuaire, s'y précipite, trouve le vieillard assoupi, le linceul et un bras du petit cadavre ont été brûlés par un cierge qui est tombé dessus. » (Freud, 1900, 433). Le fils brûle au feu du père à défaut de pouvoir le tuer in effigie, il se consume écrasé par cette présence qui l'inhibe et qu'il fuit. Le père ne peut mourir afin que le fils advienne car il ne peut le tuer. C'est bien ce que Denis répète aussi par son errance sociale et affective: il ne peut s'inscrire puisqu'il ne parvient pas à s'inscrire comme sujet différencié, identifié (il dira être un «loser»).

Denis et ses parents s'appellent au moins une fois par semaine. Comme ils partaient, Jérôme a laissé à son fils un message en lui disant: «nous allons partir en week-end et nous ne pourrons pas nous entendre, rappelle-nous ». J'ai essayé de faire entendre à monsieur ce qu'il venait de dire et me dit «en effet, je me demande si ça n'est pas depuis toujours le cas». Leur relation est fondée sur cette «mal-entente» 
mais il me semble qu'il s'agit davantage d'une tentative de Denis de sortir du désir de son propre père pour lui, désir tout-puissant pour son fils de devenir « quelqu'un ».

Jérôme a fait une carrière importante en occupant des postes de responsabilité.

La mère de Jérôme est décédée lorsqu'il avait 16 ans, et son père était un ingénieur agronome qui est devenu officier et qui tenait l'armée en haute estime, "père schreberien » s'il en est. Jérôme était en opposition à son père pour l'aspect militaire et a pris «l'armée en grippe».

Jérôme amène un rêve: «Là où je suis mais qui n'est pas ma maison, arrive un ami. Il est très mécontent car il a prêté des crayons de couleurs à Denis et voudrait les récupérer. Il me montre un objet, un crayon de couleur : "Regarde dans quel état il l'a mis.” Je vais faire mon métier de père, on va dresser une liste. »C'est la première fois que Jérôme amène un rêve en séance. Il associe sur la lecture qu'il fait en ce moment d'un roman qui s'appelle Vienne, où il est question d'un étudiant en psychiatrie. Il redit la dernière phrase du rêve en parlant de son devoir de père, ne dit plus métier et y ajoute quelques éléments. Il dresse une liste des crayons de couleurs dans le but de retrouver ce qui a été perdu mais ne trouve pas de papier pour écrire, c'est sa femme qui note sur un bloc-notes des éléments de sa biographie.

Ce rêve fait écho à la séance précédente où il était question de sa biographie et de son lien à son père... mais aussi de ce qu'il avait pu dire de son soutien financier à son fils et du règlement du loyer en cas d'insolvabilité. Dresser la liste suppose aussi de réparer les dommages commis, et cela rejoint sa préoccupation actuelle d'assurer son devoir de père en assurant ce que le fils ne peut assumer lui-même. Mais c'est aussi réparer quelque chose de leur filiation, une difficile relation assombrie par le décès du précédent fils. Jérôme souhaite dire à son fils qu'il vient me voir «pour lui » (Denis) et pense que cela l'incitera à faire lui-même un travail.

Il me dit à la séance suivante qu'il a appelé Denis afin de lui dire qu'il faisait un «chemin» avec moi «afin de réfléchir à la façon de vivre, d'être vis-à-vis de lui ». Denis n'a pas réagi sur le moment mais il a rappelé son père le soir même (alors qu’ils ne s'appellent qu'une fois par semaine) afin de lui demander s'il pouvait consommer des aliments qui avaient dépassé la date de péremption. Il opère semble-t-il une régression en se mettant sous l'autorité du père qui peut autoriser ou interdire. Il y a à entendre le lien entre le travail sur leur relation que fait le père et la consommation d'aliments («mauvais»). Ne pourrait-on pas entendre, puisqu'il s'adressait à son père à propos de la nourriture, qu'il lui demande s'il peut consommer de la relation alors que celle-ci a dépassé la date limite, qu'elle est «empoisonnée » ? Qu'est-ce qui est consommable? On sait que Freud dans Totem et tabou disait que la société de droit repose sur l'interdiction de tuer et de consommer l'animal totémique qui représente le père. Tabou vient du polynésien tapu («interdit, sacré»), il s'agit d'un système d'interdictions de caractère religieux appliquées à ce qui est considéré comme sacré ou impur.

La psychanalyse a révélé que l'animal totémique servait de substitut au père. Dans la horde originaire, il n'y avait qu'un père violent, jaloux, qui gardait toutes les femmes pour lui et évinçait les fils qui arrivaient à l'âge adulte. Un jour, les frères 
expulsés se groupèrent, abattirent et consommèrent le père, mettant un terme à la horde paternelle. Dès lors, ils parvenaient, dans l'acte de consommer celui qu'ils avaient tué, à l'identification avec lui, tout un chacun s'appropriant une partie de sa force. La double présence du père dans la scène sacrificielle : comme dieu et comme animal de sacrifice, correspond aux deux significations de la scène. «Ils haïssaient le père, qui s'opposait si violemment à leur besoin de puissance et à leurs exigences sexuelles, mais tout en le haïssant ils l'aimaient et l'admiraient. [... Le mort devenait plus puissant qu'il ne l'avait jamais été de son vivant [...].» (Freud, 1912-1913, 214)

C'est dans L'interprétation du rêve, qui paraît en 1900, que le père commence ainsi à revêtir une fonction beaucoup plus symbolique, comme si le garçon voyait dans le père, et la petite fille dans la mère, ce rival en amour, dont l'élimination ne peut que tourner à son avantage. Ces motions psychiques (amour/haine) formées dans la vie psychique enfantine sont déterminantes pour la symptomatique de la névrose ultérieure. Qu'est-ce qu'un père? C'est celui qui vient arrêter cette expérience du $u n /$ deux (l'enfant est dépendant de la mère) pour l'ouvrir au trois (le père occupant une position tierce). C'est de cet arrêt qu'autre chose se crée qui va être le lieu d'inscription des expériences futures. À partir de la constitution du sujet par le refoulement primaire et par la coupure symbolique opérée par le père, le sujet advient à la parole. Le complexe d'CEdipe est constitué par l'opération initiale de différenciation avec la mère. Ce qui la permet est l'intervention de ce que Lacan appelle le «père symbolique » ou encore «fonction de nom-du-père ». La fonction du père, dit Lacan, est d'être un signifiant substitué au premier signifiant introduit dans la symbolisation, le signifiant maternel.

Le système totémique est donc né des conditions du complexe d'CEdipe. Mais alors que Laïos, père d'CEdipe, est le père du désir, l'Urvater (père originaire) est le maître de la jouissance. Ainsi, puisque les fils étaient animés de sentiments contradictoires à l'égard du père de la horde, ils se sont livrés à des expressions d'amour exagérées qui prirent la forme du repentir. Ce que le père vivant avait empêché autrefois par le fait même de son existence, les fils se le défendaient à présent. En même temps, ils désavouaient leur acte envers le père en interdisant la mise à mort du totem. Il s'établit alors un contrat entre les fils et le père mort: le père mort promet sa protection et les fils s'engagent à ne plus l'offenser, c'est-à-dire à ne plus renouveler sur lui l'acte qui avait coûté la vie à l'Urvater. C'est au travers d'une élaboration mythique que la fonction du père a émergé dans la psychanalyse et qu'elle traverse l'œuvre de Freud jusqu'au Moïse et le monothéisme où est développée son efficacité inconsciente comme étant celle du père mort en tant que terme refoulé. Et c'est aussi le meurtre de l'Urvater qui produit la possibilité d'une réconciliation avec le père.

Lacan écrit: «[...] la nécessité l'a mené [Freud] à lier l'apparition du signifiant du Père, en tant qu'auteur de la Loi, à la mort, voire au meurtre du Père, — montrant ainsi que si ce meurtre est le moment fécond de la dette par où le sujet se lie à vie à la Loi, le Père symbolique en tant qu'il signifie cette Loi est bien le Père mort.» (Lacan, 1966, 556) 
Denis rappelle encore son père le matin suivant pour lui dire qu'il irait aujourd'hui à l'ANPE (alors qu'il s'enferme chez lui depuis de nombreux mois ne souhaitant et ne pouvant exercer aucune activité). Il lui dit encore qu'il a fait, la nuit suivant l'appel de Jérôme, un curieux rêve: «Je marchais dans une curieuse rue comme la rue Mouffetard, je voyais des taureaux, un troupeau de taureaux, l'un d'eux s'est détaché du groupe et m’a foncé dessus. » Sur ce récit de rêve raconté au père, il dit: «On ne peut être et avoir été.» Ce rêve, comme ce que me raconte depuis le début de séances Jérôme, me fait penser que Denis est à la fois très fragile et saisi par des émotions d'une grande violence qui semblent se retourner contre lui-même. Dans quelle mesure est transposé dans la filiation de Jérôme avec ses fils, ce que lui en tant que fils n'a pu symboliser dans sa relation à ce père militaire qu'il prend en grippe, donc là où quelque chose se grippe. À ne pas vouloir lui ressembler, ne lui donne-il pas une vraisemblance imaginaire ${ }^{2}$ ? Avec quoi Denis se débat-il ? Quels sont les taureaux qui le menacent? Quelle(s) figure(s) hante(nt) ainsi Denis au point de rester enfermé chez lui?

Le complexe de castration est le plus fort traumatisme que subit le jeune garçon et Freud renvoie la terreur provoquée par cette menace à une trace mnésique phylogénétique (violence du père primitif). Autrement dit la mise à mort du père primitif et sa consommation resteraient présent dans la mémoire du sujet. Dans notre exemple, on a l'impression que le père mort aurait été gelé, gardé au frais en attendant sa consommation. En somme la question qui se pose pour ce fils est celle de savoir s'il peut consommer le père après-coup (après sa date de péremption), s'identifier à lui (chercher du travail à l'ANPE ${ }^{3}$ ) là où il n'a cessé d'être dans la transgression se faisant renvoyer de l'école puis des autres institutions. Il va à l'ANPE après avoir fait ce rêve et l'avoir raconté à son père. Consommation d'aliments périmés, angoisse de castration et désir de se réinsérer professionnellement. On voit bien cette errance identitaire, il ne peut s'inscrire comme sujet du nom. Pour paraphraser ce que dit Lacan à propos de Joyce, on pourrait dire que Denis reste enraciné dans son père tout en le reniant.

Un nom est donné à la plupart des enfants, mais ce n'est pas parce qu'ils le reçoivent qu'ils le prennent. Pour que ce nom soit pris, un combat avec le père s'ordonne, et de l'issue de cette lutte dépend l'adéquation du sujet à son corps de jouissance. Si le nom inscrit d'abord le sujet comme chaînon intermédiaire dans la suite des générations, ce nom, en tant que signifiant intraduisible, supporte et transmet le refoulement et la castration symbolique. Freud dit qu'il faut dénoncer le caractère magique et totalisant des noms afin que le nom du père puisse être hérité par le fils.

Dans son séminaire sur Les Non-Dupent-Errent ${ }^{4}$, Lacan énonce que «si, mythiquement, le père ne peut être qu'un animal, le père primordial, le père d'avant l'interdit de l'inceste ne peut être avant l'avènement de la culture, et conformément au mythe de l'animal sa satisfaction est sans fin; le père est ce chef de horde. Mais qu'il l'appelle Totem, et justement à la lumière des progrès apportés par la critique de l'anthropologie structurale de Lévi-Strauss qui met en relief l'essence classificatoire du Totem, ce qu'il faut en second terme, c'est mettre au niveau du père la fonction du nom. » 
Jérôme vient me voir après une semaine d'interruption et rapporte ce rêve qui, me dira-t-il en fin de séance fait écho au rêve de son fils. Il souhaitait le lui raconter mais « on s'est raté » (à entendre comme on n'a pas pu se joindre au fil). «Je suis seul dans une réunion mondaine. Je quitte cette salle, m'en vais. Je tombe nez à nez avec trois jeunes femmes qui me disent «vous partez sans me dire au revoir». Je traverse une propriété, sors par une petite porte, je vois arriver sur ma droite trois personnages plutôt de petite taille, qui ont un casque sur la tête. Le premier a un casque allemand sur la tête, petit bonhomme qui me regarde, me parle. J'ai un marteau à la main, je le menace. Le personnage derrière est un bonze qui lui adresse la parole et chacun part de son côté. » Il associe ainsi : le petit bonhomme, c'est son fils, les trois jeunes femmes sont ses trois filles et le bonze c'est la psy. Il dit que son fils se protège par le casque. Ce rêve vient répondre au rêve du fils qui se trouvait poursuivi par le taureaupère.

Lorsque Denis appelle, il téléphone au domicile parental (ligne fixe), jamais sur les portables de son père ou de sa mère. Il appelle souvent pour sa santé («j’ai mal aux oreilles, que dois-je faire?») ou alors parce qu'il n'a pas suffisamment de quoi s'acheter à manger ( «c'est dur de ne pas toujours manger à sa faim»). Jérôme me dit qu'il l'a appelé «parce qu'il criait famine». On peut supposer une régression de type psychotique. Denis appelle à l'aide dans ce cri de détresse, et c'est malgré tout vers les parents qu'il se tourne.

Après quelques semaines d'interruption des séances en raison des vacances, Jérôme me dit qu'il y a du nouveau puisque son fils a décidé de «se faire soigner ». Il semblerait que Denis soit dans un état de dépression grave et demande de l'aide à sa famille. Dans un premier temps il me dit qu'avant qu'il ne manifeste cette détresse, Jérôme dit à son fils que s'il décidait de voir un psy comme lui, il envisagerait de diminuer ses propres séances (une par mois au lieu de quatre) afin de lui verser cette somme (celle de ses séances) pour assurer sa psychothérapie. J'ai réagi en disant à Jérôme qu'il confondait les espaces et que son fils n'avait pas à dépendre de son argent, et encore moins celui consacré à sa propre analyse pour entreprendre un travail qui justement viserait à se « séparer» de son propre père. Il me semble que Denis soit sous cette dépendance et que son père ne fait que l'accentuer. Jérôme pensait que si Denis voulait se faire soigner là où habitent ses parents, il pourrait même envisager qu'il réside dans leur maison et dans ce qui fut sa chambre d'enfant.

Jérôme me parle de ce qui identifie son fils sur internet (messagerie «XXX») : «Loser». Le travail que fait monsieur en analyse rend possible une écoute et un autre positionnement auprès de son fils, si bien que ce dernier arrive beaucoup mieux à parler à son père «là où autrefois c'était bonjour, bonsoir». Denis sent un véritable soutien de la part de son père. Celui-ci lui a proposé de venir l'aider à faire ses papiers qu'il ne parvient pas à faire seul, mais Denis préfère aller chez son père car, dit-il, «j'ai besoin de me retirer à tout moment». La proximité avec ses parents semble anxiogène, il évite le contact (tout rapprochement), leur parle parfois par répondeur interposé. Toutefois, depuis le début de la thérapie de Jérôme et suite au rêve du taureau, Denis semble demander de l'aide et être d'accord d'aller lui aussi vers un 
thérapeute. Son père a donc cherché un $\mathrm{CMP}^{5}$ proche de chez lui et il a accepté de s'y rendre.

La semaine suivante, Jérôme m'annonce une "demi-réussite»: son fils s'est rendu deux fois devant le CMP mais n'a pu franchir le seuil. Je lui fais entendre que justement, il est bien question d'une difficulté de seuil, lui-même étant dans un état de seuil, dans un entre-deux (il dit bien qu'il n'est pas encore adulte, mais un enfant dans un corps d'adulte).

Jérôme amène un rêve qui me semble être le pendant du rêve du taureau du fils. "Je suis dans la rue, je vois un char qui avance dans la rue; à un moment, il oblique et il entre dans une maison. Il n'y a pas beaucoup de fêlures dans la maison malgré cet événement. Ce n'est pas possible un char qui entre dans une maison, il faut avertir un responsable. Puis je vois une scène où je suis chez moi et il se trouve que j'ai une boule de merde dans les mains. Ma femme m'appelle, je ne peux la voir comme cela alors je la jette dans une corbeille. » Jérôme associe sur le fait qu'il aurait aimé se débarrasser d'une situation «emmerdante» avec sa femme. En fait, tous deux ne sont pas d'accord sur la manière de s'y prendre avec Denis et Jérôme aimerait se faire entendre de toute la famille comme ayant maintenant à s'imposer (le char) face à toutes ses femmes (sa femme et ses quatre filles) sans faire de dégâts ( «il n’y a pas de fêlures»). Ce père qui est physiquement impressionnant (il me dit d'ailleurs qu'on lui fait remarquer à quel point il est impressionnant, intimidant) n'est pas aussi autoritaire qu'il en a l'air, mais il donne à voir de la «machine» phallique. J'ai aussi été vivement impressionnée par ce personnage lorsqu'il est arrivé la première fois dans mon cabinet. Il est très grand, très imposant, et donnant d'une voix très grave et forte.

Le dispositif que nous avons mis en place donne une place à Denis qui semble s'en saisir. Sa tentative d'approche du lieu de «soin» choisi par son père est une approche vers nous. Denis semble aller beaucoup mieux, il dit avoir pu ranger ses papiers et il a abordé avec son père sa relation à sa mère. Il lui a dit: "C'est lié à la mort de J. (son frère mort à l'âge de quatre ans, deux ans avant sa naissance). Je pense que c'est pour cela que maman n'a pu avoir vis-à-vis de moi l'affection qu'elle aurait dû avoir.»C'est la première fois que Denis s'exprime ainsi à son père, il est plus ouvert et moins craintif de lui parler car Jérôme est aussi plus à l'écoute et se sent soutenu par notre dispositif. Jérôme me dit que sa femme n'a pas eu d'affection de sa propre mère et qu'elle n'a pas pu en manifester auprès de Denis ( « ma femme a manqué de tendresse maternelle») ; ce dernier s'étant senti exclu enfant, il rejoue actuellement cette place d'exclu à l'égard de la société et de sa famille (précarité, sans travail, dépendant des versements de ses parents).

Il me semble que Denis n'est pas inscrit au registre des vivants. Il est un mortvivant n'arrivant pas à prendre une place. Le travail de thérapie de monsieur met en mouvement toute la famille et les fait travailler autour de ce pôle qui finalement les rassemble en les inquiétant. Les secrets et les affects émergent et amènent la parole. On peut être né physiquement mais pas psychiquement: Denis dit d'ailleurs qu'il n'est pas devenu adulte, ni vivant ni mort, comme suspendu en attente d'une 
autorisation de résidence à la vie. Comme le dit Antoine dans le Jules César de Shakespeare (III, 2, v. 105) «My heart is in the coffin there with Caesar. » Ce que l'on peut traduire ainsi : «Mon cœur est là dans le cercueil avec César. » Le mort emporte avec lui le naissant qui le suit et c'est comme si le nouveau-né ne pouvait pas prendre corps, s'inscrire au sein d'une famille prise par un mort qui ne cesse de ne pas mourir. Denis semble porter le plus gentil que lui, il est un être comparatif. Il me semble qu'en transgressant les « règlements intérieurs » (rappelons-nous qu'il ne cessait de faire l'école buissonnière et qu'il se présentait toujours comme un "mauvais garçon » ayant des potentialités certaines) il a tenté de faire sa vie mais qu'il a dû être rattrapé par le poids du mort. C'est ce qui fait dire à son père qu'il est peut-être maniaco-dépressif car il a eu des périodes d'enthousiasme professionnel. Ce sont simplement des tentatives de se tirer des affaires du mort. Denis semble arrêté dans le temps, ralenti, en sursis, coupé du monde. La thérapie a permis la création d'un dialogue entre père et fils, et la possibilité pour Denis de lui parler a relancé ce processus de retour à la vie. "La renaissance suit sont cours » me dit Jérôme à la séance suivante. Denis lit son courrier et dit à son père que «c'est merveilleux de se sentir sortir de cette dépression ». Le fils souhaite me rencontrer; je fais part à Jérôme de l'importance de ce désir même si je ne souhaite pas pour le moment le recevoir. Denis aurait dit que s'il y avait encore quelque chose qu'il souhaiterait voir améliorer, c'est sa relation à sa mère.

Le père rêve d'un univers clos, est-ce un hôpital ou alors une croisière dans un bateau. Un personnage lui parle, c'est un homme de grande allure, qui dans le rêve s'appelle Le Cid, et ce dernier lui parle d'un enfant ou d'un adolescent. Le Cid s'interroge sur ce qui va se passer avec le psy. Jérôme trouve que c'est un personnage avec beaucoup de panache, c'est une figure paternelle. Dans la pièce de Corneille, Le Cid est un surnom de guerre, c'est un vainqueur qui venge son père pour conquérir Chimène. Un autre rêve l'amène aussi à figurer un homme politique de grande envergure. Jérôme aurait-il le désir que son fils prenne de l'étoffe afin de lui ressembler et conquérir ainsi les femmes qui lui permettraient d'assurer une descendance, une filiation? Craindrait-il aussi que son fils, par ce travail, devienne plus fort que lui et se venge?

Il faut tuer le père symboliquement pour vivre, s'en affranchir au risque de demeurer enfant rebelle. Denis se révolte contre le père mais ne le tue pas symboliquement et donc, se sent menacé par lui : le rêve du taureau figure toute la puissance du père et rend dès lors possible l'affrontement pour une mise à mort (ce rêve rappelons-le a été l'amorce d'un travail avec Jérôme du lien père-fils). Le travail analytique permet la mise en place du Nom-du-Père de sorte que le sujet puisse s'en passer à condition de s'en servir. Denis est aux prises avec les processus adolescents en refusant la destitution du père œdipien. Le soutien du père œdipien est un refus du passage adolescent, un refus du renoncement à la promesse œedipienne infantile d'une puissance à venir. Le père imaginaire reste alors un modèle vivant que le sujet peut égaler, espérant ainsi reprendre à son compte, ou pour son usage, la puissance qu'il lui a attribuée au temps de l'enfance. 
Comme le dit déjà Freud (1912-1913), le père est tué in effigie, en image, on brise sa statue au risque de ne plus exister soi-même. Le mythe de la horde primitive de Totem et tabou est bien un mythe. Le meurtre du père est un passage obligé pour tout sujet quand il doit s'inscrire dans le groupe social au-delà de son appartenance au groupe familial, bref quand il devient adulte et joue ailleurs que dans la famille les enjeux du désir et de la satisfaction. Ce meurtre nécessaire du père est au cœur des processus adolescents quand le sujet s'affronte à la question de la construction de sa paternité. On voit bien à quel point il était impossible pour Denis de créer une «alliance» avec une femme et de devenir père. Le père imaginaire de l'Gdipe doit être destitué de ses fonctions de père tout-puissant interdisant l'accès à la mère du désir pour l'enfant, le soulageant ainsi de la charge de cette jouissance maternelle. Ce père ne peut continuer à exister pour le garçon devenant adulte. Sa statue (rappelonsnous la figure du taureau: identification du père à l'animal totémique) doit tomber pour permettre au garçon de l'égaler et de conquérir d'autres femmes. Ce mouvement qui fait du père-héros un pauvre type est le propre même de l'adolescence (il s'agit bien du père œdipien imaginaire). Pour Denis, la mise à mort de ce père-là était jusqu'alors impossible, il s'est retiré du combat pour se réfugier dans un premier temps dans la transgression puis dans la dépression (ce qui a pu aussi par moment aller ensemble). Nous ne pouvons pas dire que Jérôme était un père défaillant mais qu'au contraire l'image qu'il donne à voir (très imposant et intimidant aux yeux de proches) est telle qu'il sidère probablement le fils de toute action ${ }^{6}$.

Il faut donc que le père des origines soit démantelé pour advenir à la fonction d'adulte (homme et père). La part de l'infantile est restée en panne chez Denis, d'où la difficulté du passage adolescent et sa dimension de meurtre du père. Il me semble que Denis est resté sidéré devant cette figure, happé par une image qui l'a fait fuir à défaut de s'y confronter. Jérôme qui n'a rien d'un père autoritaire mais simplement l'aspect; le physique ne viendrait-il pas incarner l'uniforme du grand-père (militaire et autoritaire), la cuirasse inquiétante qui fige et empêche tout rapprochement? Une figure est portée sur une autre et densifie son aspect qui le rend impressionnant. Et c'est bien sur cet aspect que Denis bute depuis l'enfance en se construisant contre cette autorité.

Nous pouvons apporter quelques précisions ou éclairages quant aux différents registres de la paternité tels qu'ils sont amenés par Lacan qui distingue père réel, père imaginaire et père symbolique. Nous avons évoqué le père imaginaire de l'Édipe, père rival. Le père est à la fois objet d'une identification primaire, pris d'emblée comme idéal et comme rival pour le garçon lorsqu'il tente de s'approprier le premier objet d'amour qu'est la mère. Le père réel est celui de la réalité familiale dont on attend qu'il fasse valoir la loi symbolique (interdit de l'inceste). Il est celui qui permet à l'enfant d'avoir accès au désir sexuel et d'avoir une position virile (on voit bien dans notre exemple que ce père-là est défaillant puisque DENIS n'a jamais pu tenir cette place auprès d'une femme). Le père symbolique est celui auquel renvoie la loi (l'interdit étant proféré au Nom-du-Père), il s'agit du père mort (meurtre du père de la horde primitive). La castration, comme opération qui limite le désir, n’est pas à 
confondre avec des mutilations réelles mais pourtant cet imaginaire est présent chez le sujet, d'autant lorsque la castration symbolique n'a pas opéré (voir le rêve du taureau de X.).

Ce cas clinique n'est pas sans m'évoquer la magnifique et poignante lettre de Kafka (1919) à son père. "Tu m’as demandé l'autre jour pourquoi je dis que je te crains. Comme d'habitude je n'ai rien su te répondre, en partie justement à cause de la crainte que j'ai de toi [...]», «[...] je ne te crois absolument pas coupable du fait que nous sommes devenus étrangers l'un à l'autre. [...] quelque chose ne va pas entre nous et que tu y as contribué, mais sans être coupable. ». "Mais comme père précisément tu étais trop fort pour moi, d'autant que mes frères étaient morts en bas âge, que les sœurs sont venues bien plus tard et qu'il m'a donc fallu soutenir tout seul le premier choc, ce pour quoi j'étais beaucoup trop faible.» (p. 11-14)

Il évoque la force, la santé, la puissance vocale de son père mais aussi ses méthodes d'éducation: «[...] j’étais encore torturé à la pensée que ce géant, mon père $[. .$.$] était capable, presque sans raison, de venir la nuit me sortir du lit pour me$ porter [dehors] et que j'étais donc un tel rien pour lui. ». Il est dominé par un sentiment de nullité qui tient à l'influence de son père. Ce dernier le félicitait quand il marchait au pas «mais je n'étais pas un futur soldat». Il se sentait écrasé par son apparence physique, par ses cris qui étaient comme un commandement du ciel. « [...] tu m'as très tôt interdit la parole ». Il décrit une mère d'une «bonté sans limites » mais qui l'empêchait par là même de s' «arracher» du père puisqu'elle se faisait son avocate et le « rejetait dans son cercle» (p. 17-18, 27).

Kafka perdit toute confiance en lui en sa présence. Il parle de l'aversion du père pour son «travail d'écriture»: «[...] $j$ 'avais fait un bout de chemin autonome pour m'éloigner de toi, même si cette avancée rappelait un peu le ver de terre qui, l'arrière du corps coincé sous le pied qui l'écrase, s'arrache le devant et se traîne à l'écart.». Le « poids» de son père le tirait toujours avec plus de force vers le bas. « La conscience de ma valeur dépendait beaucoup plus de toi que de n'importe quoi d'autre [...].». L'écriture a opéré comme une possibilité de devenir, de se tenir debout face au père, de fixer l'angoisse, essentiellement dans ce que Deleuze appelle un devenir-animal (notamment pour ses nouvelles) (p. 60, 65).

Jérôme a été hospitalisé pour un problème assez sérieux (AVC) et n'a pas souhaité reprendre sa thérapie. Monsieur a un certain âge qui ne le met pas à l'abri de ce genre d'accidents, toutefois je me suis posée la question de ce qui a surgi pour lui dans le réel du corps à ce moment-là. Quel est cet arrêt brutal suite à cette hospitalisation? Alors que pendant son séjour à l'hôpital, il m'a appelée afin de me dire qu'il avait rêvé et qu'il lui faudra me raconter ses rêves.

Par téléphone, il a pu dire que sa relation à Denis s'était transformée et que ce dernier avait souhaité «se prendre en main». Denis souhaiterait faire un travail sur lui mais il a besoin encore d'un peu de temps avant de franchir le pas (de la thérapie). Il me semble que le rêve du taureau a été déterminant car il signifiait aussi que Denis se faisait entendre dans la séance et qu'il a pu évoquer à son père, par ce rêve, qu'il le sentait imposant et rigide. 
Ce dispositif thérapeutique pose de nombreuses questions. Entre autres, celle de la construction en analyse ${ }^{7}$. Construire avec des souvenirs un passé, c'est retrouver des moments significatifs d'une construction psychique. La construction que fait l'analyste pour en rendre compte est conjecturale. Cette construction se fait à deux, l'analyste et l'analysant voire même comme dans cet exemple, un proche de l'analysant absent s'impliquant dans l'analyse par la production de formations de l'inconscient rapportées en séance par l'analysant lui-même. Cet espace de construction des pensées crée l'espace analytique. Je pense que la présence (absence) d'un tiers actif/impliqué rêvant pour l'analyste doit être pensée dans le sens où elle contribue à la construction de l'espace analytique (nous pourrions évoquer le cas du «petit Hans» où Max Graf, père du petit garçon de cinq ans, dirige, sous le contrôle de Freud, l'analyse de son fils Herbert). Il s'agit alors d'inventer du possible avec du fragile et de l'incertain, avec des paroles croisées entre père et fils. Il me semble que ces séances ont permis que s'instaure un espace de jeu (je) entre père et fils, un espace où l'imaginaire se déploie au fil des générations (de pères). Je mesure que cette construction est inachevée du fait même de l'interruption des séances et qu'elle est très certainement insuffisante mais il m’a semblé malgré tout intéressant de poser quelques séquences assez éclairantes de ce malaise entre père et fils.

Céline Masson

3 allée Jean Giraudoux 78420 Carrières-sur-Seine celine.masson@free.fr

\section{Notes}

1. Je lui fais entendre «le» fils, il me dit « "mon" fils si vous préférez».

2. Je remercie Xavier Gassmann d'avoir réfléchi sur ce cas et d'y avoir apporté des éclairages intéressants.

3. Agence nationale pour l'emploi.

4. Le Séminaire livre XXI (1973-1974), Les Non-Dupes-Errent, inédit, version de l'A.L.I., p. 422. (document Word).

5. Centre médicopsychologique.

6. Et peut-être bien, mais là n'est qu'une possibilité interprétative, la mort de l'enfant précédent en plein Edipe aurait pu faire craindre encore davantage ce père comme un père non seulement castrateur mais meurtrier; rappelons que Denis est né deux ans après la mort du frère décédé alors qu'il avait 4 ans.

7. Voir S. Freud, 1937, 269-281. La leçon clinique de ce texte est que le symptôme ne se laisse saisir dans le processus analytique que comme séquence de sens avec quoi il est possible de construire.

\section{Références}

FREUD, S., 1900/1987, L'interprétation des rêves (Die Traumdeutung), G. W, II-III, 1-642, trad. Ignace Meyerson, P.U.F., Paris, $6^{\mathrm{e}}$ tirage.

FREUD, S., 1912-1913/1965, Totem et tabou - Interprétation par la psychanalyse de la vie sociale des peuples primitifs, (Totem und Tabu), G.W., IX, trad. Serge Jankélévitch, Payot, Paris.

FREUD, S., 1937/1985, Constructions dans l'analyse (Konstruktionen in der Analyse), in Résultats, idées, problèmes II, 1921-1938, PUF, Paris. 
KAFKA., F., 1919/1994, Lettre au père, Éditions Ombres, Toulouse.

LACAN, J., 1966, D’une question préliminaire à tout traitement possible de la psychose, Écrits, Seuil, Paris.

LACAN, J., inédit, Le Séminaire livre XXI (1973-1974), Les Non-Dupes-Errent, version de l'A.L.I., (document Word). 